\title{
Application of the linearly independent numerical manifold method in modeling the complex crack problems
}

\author{
Dong-Dong $\mathrm{Xu}^{1}$, Xiao-Bing Wang \& Yu-Jie Sun ${ }^{2}$ \\ ${ }^{1}$ Key Laboratory of Geotechnical Mechanics and Engineering of Ministry of Water Resources, \\ Yangtze River Scientific Research Institute, Wuhan 430010, China \\ ${ }^{2}$ Sinopec Petroleum Engineering Design Limited Company, Dongying 257026, China
}

\begin{abstract}
KEYWORD: Numerical manifold method; complex crack problems; linear dependence; mathematical cover; physical cover; manifold element

ABSTRACT: Numerical manifold method (NMM) is very suitable for modeling the transition from continuum to discontinuum by virtue of its advanced finite cover technique. Compared with the 0 -order NMM, higher-order displacement functions are more suitable for modelling the crack problems as the result that the latter usually shows higher precision than the former under the same mesh density. However, the higher-order NMM may be suffering with the linear dependence problems, such as the 1-order NMM which adopts 1-order polynomials as its cover functions. Xu et al. (2015) has proposed a new higher-order NMM which has no linear dependence problems and has been applied to solve simple crack problems. In the paper, it is applied to solve the complex problems such as the multiple branched and intersecting cracks in order to show its advantageous features. The excellent results show that the proposed method is also excellent in even treating the complex problems.
\end{abstract}

\section{introduction}

In recent years, the modeling of transition from continuum to discontinuum has been a hot topics. In real world, the shapes of crack are usually very complex. The modeling of complex crack problems must be more important for the researchers and engineers to predict the life span of cracked structures under service conditions (Daux et al. 2000, Ma et al. 2009). Several numerical methods have been applied to solve the complex problems.

Finite element method (FEM) introduces the interference element or contact element to model the crack problems (Trädegård et al. 1998, Bouchard et al. 2003). One major shortage of the FEM is that the mesh must be accordance with the crack. The meshless (or element-free) method (Belytschko et al. 1996) is no longer suffering with the accordance problems as in FEM, because the problem domain is covered by some nodes and no element is generated. However, it usually has some difficulties in the numerical integration and applying the essential boundary conditions (Strouboulis et al. 2000). The extended finite element method (XFEM) (Dolbow \& Belytschko 1999) and generalized finite element method (GFEM) (Strouboulis et al. 2000) can be viewed as the advanced FEM and they are all within the framework of partition of unity method (PUM) (Melenk \& Babuška 1996). The introduced generalized Heaviside functions and enriched functions make XFEM more suitable for modeling complex problems than FEM and there is no need to make crack and mesh be accordance with each other. GFEM is the same to NMM in essence because the order of local displacement functions can be selected freely.

NMM (Shi 1991) proposed by Shi has realized to solve the continuous and discontinuous problems in a unified way based on its advanced finite cover technique. NMM has been tried to solve the crack problems (Zhang \& Zhang 2012). As known, the 1-order NMM is suffering with the linear dependence problems. In order to overcome this shortage, $\mathrm{Xu}$ et al. (2015) has proposed a new higher-order NMM, which is denoted as NMM-P in this paper because the local displacement functions are derived from the triangular plate element of FEM (Zhu 1998). The NMM-P has shown good features in solving the simple crack problems. In the paper, it is further applied to solve the complex problems such as the multiple branched and intersecting cracks in order to exhibit its advantageous features. 


\section{THE FOUNDATIONS OF NMM-P}

NMM is based on the mathematical cover (MC), physical cover (PC) and contact loop. We take triangular mesh as the mathematical cover in the paper. So the union of the 3 triangles sharing the same node is defined as a mathematical patch (MP). Furthermore, the union of all mathematical patches is defined as the MC, which should cover the whole problem domain. Physical patches (PP) are generated by intersecting all the components of problem domain with mathematical patches. All the physical patches form the physical cover. The common region of as more as possible physical patches is defined as the manifold element (ME). The contact loop is used to model the contact behaviors between different loops. More details about NMM can be found in the references (Xu et al. 2015).

The physical patch where the crack tip locates is defined as the singular physical patch, while the others are defined as non-singular physical patches. Based on the classification, the manifold elements are therefore can be classified into three types: 1) normal manifold element (covered by only non-singular physical patches), mixed manifold element (covered not only by non-singular physical patches but also singular physical patches) and the singular manifold element (covered by only singular physical patches).

For the sake of completeness, the local functions of NMM-P are restated in the paper. At first, we assume that the interest manifold element is covered by 3 physical patches denoted as PP- $i$, PP- $j$ and PP- $m$. For the non-singular physical patch PP- $i$, its local displacement functions $\boldsymbol{w}_{i}(x, y)$ are defined as follows

$$
\boldsymbol{w}_{i}(x, y)=\boldsymbol{T}^{i}(x, y) \boldsymbol{d}_{i}
$$

$\boldsymbol{T}^{i}$ are the base functions, $\boldsymbol{d}_{i}$ are the degrees of freedom (DOFs). Their expressions are as follows

where

$$
\begin{gathered}
\boldsymbol{T}^{i}(x, y)=\left[\begin{array}{cccccc}
N_{i} & 0 & -N_{i y} & 0 & \frac{1}{2} N_{i x} & \frac{1}{2} N_{i x} \\
0 & N_{i} & 0 & N_{i x} & -\frac{1}{2} N_{i y} & \frac{1}{2} N_{i y}
\end{array}\right] \\
\boldsymbol{d}_{i}^{T}=\left(\begin{array}{llllll}
u^{i} & v^{i} & \varepsilon_{x}^{i} & \varepsilon_{y}^{i} & \gamma_{x y}^{i} & \omega^{i}
\end{array}\right)
\end{gathered}
$$

$$
\begin{gathered}
N_{i}(x, y)=1+L_{i} L_{j}+L_{i} L_{m}-L_{j}^{2}-L_{m}^{2} \\
N_{i x}(x, y)=b_{j} L_{i} L_{m}-b_{m} L_{i} L_{j}+\frac{1}{2}\left(b_{j}-b_{m}\right) L_{j} L_{m} \\
N_{i y}(x, y)=c_{j} L_{i} L_{m}-c_{m} L_{i} L_{j}+\frac{1}{2}\left(c_{j}-c_{m}\right) L_{j} L_{m} \\
b_{j}=y_{m}-y_{i}, \quad b_{m}=y_{i}-y_{j} \\
c_{j}=x_{i}-x_{m}, \quad c_{m}=x_{j}-x_{i}
\end{gathered}
$$

where $L_{i}, L_{j}$ and $L_{m}$ are the weight functions corresponding to PP-i, PP-j and PP-m. $\quad\left(x_{i}, y_{i}\right)$, $\left(x_{j}, y_{j}\right)$ and $\left(x_{m}, y_{m}\right)$ are the coordinates of interpolation points of the 3 physical patches. $u^{i}$ and $v^{i}$ are the rigid body displacements, $\varepsilon_{x}^{i}, \quad \varepsilon_{v}^{i}$ and $\gamma_{x y}^{i}$ are the strain components, $\omega^{i}$ is the rigid body rotation angle.

The local displacement functions on PP- $j$ and PP- $m$ can be obtained in the same way as that of PP- $i$.

PP- $i$ must be singular if there is a crack tip in the interior of it, so an enriched functions $\boldsymbol{w}_{i}^{\mathrm{s}}$ used for modeling the stress singularity around the crack tip should be added, which can be expressed as

$$
\boldsymbol{\Phi}^{i}=\left[\begin{array}{cccccccc}
\Phi_{1} & 0 & \Phi_{2} & 0 & \Phi_{3} & 0 & \Phi_{4} & 0 \\
0 & \Phi_{1} & 0 & \Phi_{2} & 0 & \Phi_{3} & 0 & \Phi_{4}
\end{array}\right]
$$


$\left(\begin{array}{llll}\Phi_{1} & \Phi_{2} & \Phi_{3} & \Phi_{4}\end{array}\right)=$

$$
\left(\sqrt{r} \cos \frac{\theta}{2} \quad \sqrt{r} \sin \frac{\theta}{2} \quad \sqrt{r} \cos \frac{3 \theta}{2} \quad \sqrt{r} \sin \frac{3 \theta}{2}\right)
$$

where $(r, \theta)$ is the polar coordinate system as shown in Figure 1 and $s_{i}$ is the degrees of freedom corresponding to the singular base functions $\boldsymbol{\Phi}^{i}$.

Here, the NMM which adopts 1-order polynomials as the displacement functions on the physical patch is denoted as NMM-1.

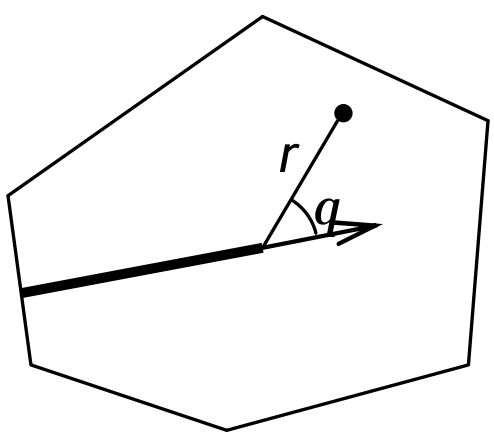

Figure 1. Polar coordinate system

\section{NUMERICAL EXAMPLES}

\section{A crucifix crack in a finite square plate}

As shown in Figure 2, there is a crucifix crack in a finite square plate suffering with bi-axial tension, which is obviously a mode I intersecting crack problem. The corresponding parameters are as follows: $\sigma=1.0, W=0.5, a=0.1,0.15 \ldots, 0.35$. The mathematical cover adopted when the crack length is 0.35 is shown in Figure 3 and the corresponding physical patches, manifold elements and degrees of freedom for all the cases are shown Table 1 . Number of the mathematical patches is fixed as 2916. It is easy to see that with the increase of the crack length, the crack will intersect with more mathematical patches, so the numbers of corresponding physical patches and manifold elements will increase. The degrees of freedom include two parts: 1) the degrees of freedom corresponding to the non-singular base functions; 2) the degrees of freedom corresponding to the singular base functions. The maximum value of PPs, MEs and DOFs are 3062, 5778 and 18468 respectively.

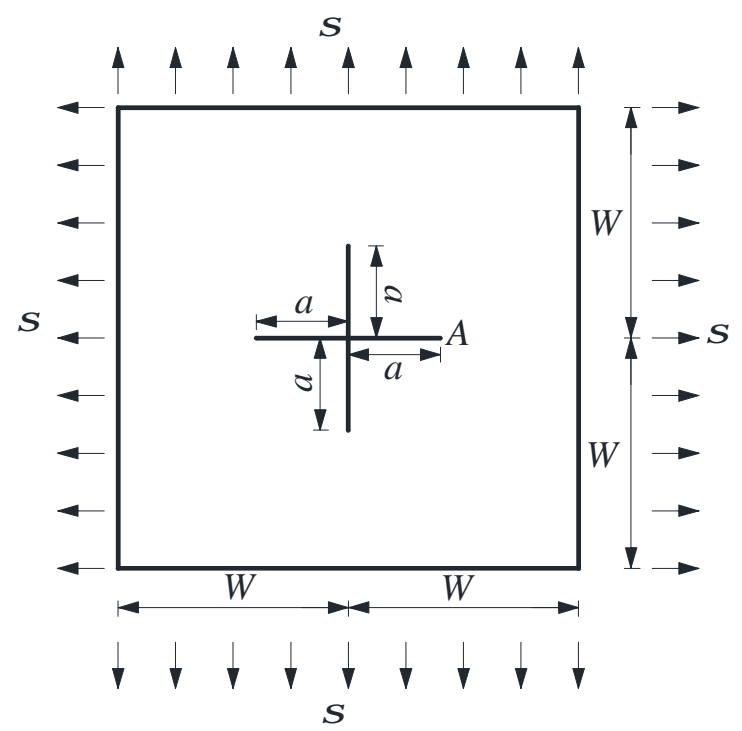

Figure 2. A finite square plate with a crucifix crack under bi-axial tension. 


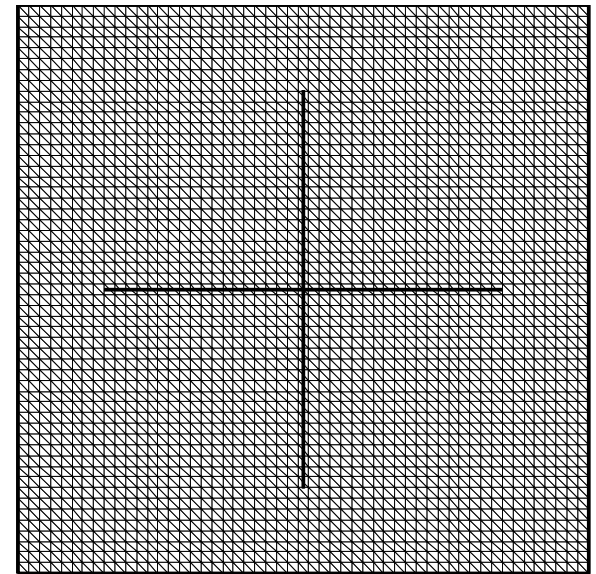

Figure 3. The mathematical cover adopted for the crucifix crack when crack length is 0.35 .

Table 1 Number of physical patches, manifold elements and degrees of freedom for different crack lengths

\begin{tabular}{lllllll}
$a$ & 0.1 & 0.15 & 0.2 & 0.25 & 0.3 & 0.35 \\
\hline PPs & 2954 & 2974 & 2998 & 3018 & 3038 & 3062 \\
MEs & 5670 & 5690 & 5714 & 5734 & 5754 & 5778 \\
DOFs & 17820 & 17940 & 18084 & 18204 & 18324 & 18468 \\
\hline
\end{tabular}

Table 2 Comparison of normalized SIFs $F_{\mathrm{I}}^{A}$ at crack tip A with different crack length $a$

\begin{tabular}{lllllll}
\hline$a$ & 0.1 & 0.15 & 0.2 & 0.25 & 0.3 & 0.35 \\
\hline NMM-P & 0.8841 & 0.9098 & 0.9519 & 1.0203 & 1.1330 & 1.3277 \\
NMM-1 & 0.8827 & 0.9079 & 0.9504 & 1.0189 & 1.1316 & 1.3264 \\
$*$ & 0.8840 & 0.9131 & 0.9575 & 1.0281 & 1.1331 & 1.2863 \\
$* *$ & 0.8800 & 0.9092 & 0.9537 & 1.0223 & 1.1300 & 1.2866 \\
$* * *$ & 0.8844 & 0.9147 & 0.9572 & 1.0253 & 1.1348 & 1.3170 \\
\hline
\end{tabular}

*Zhang \& Zhang (2012), ** Cheung et al. (1992), ***Daux et al. (2000)

The normalized stress intensity factors (SIFs) at crack tips $A$ is defined as follows

$$
F_{\mathrm{I}}^{A}=K_{\mathrm{I}}^{A} /(\sigma \sqrt{\pi a})
$$

As shown in Table 2, the normalized SIFs by NMM-P and NMM-1 are nearly the same for all the cases. Meanwhile, they are also very close the three reference results and the maximum relative error is nearly within $1 \%$ when the crack length is less than 0.3 . While the relative errors between the results by NMM-P and the three reference results are $3.22 \%, 3.19 \%$ and $0.81 \%$ respectively when the crack length is equal to 0.35 , which means that the results by NMM-P are more close to that by XFEM (Daux et al. 2000).

\section{A star-shaped crack in a finite square plate}

A star-shaped crack in a finite square plate subjected to bi-axial tension is examined as shown in Figure 4. The length of square plate is $2 W=4$ and the crack length is denoted as $a$. The mathematical cover adopted for the star-shaped crack when $a / W=0.5$ is shown in Figure 5. The normalized SIFs at crack tips with different ratios $a / W$ are discussed. 


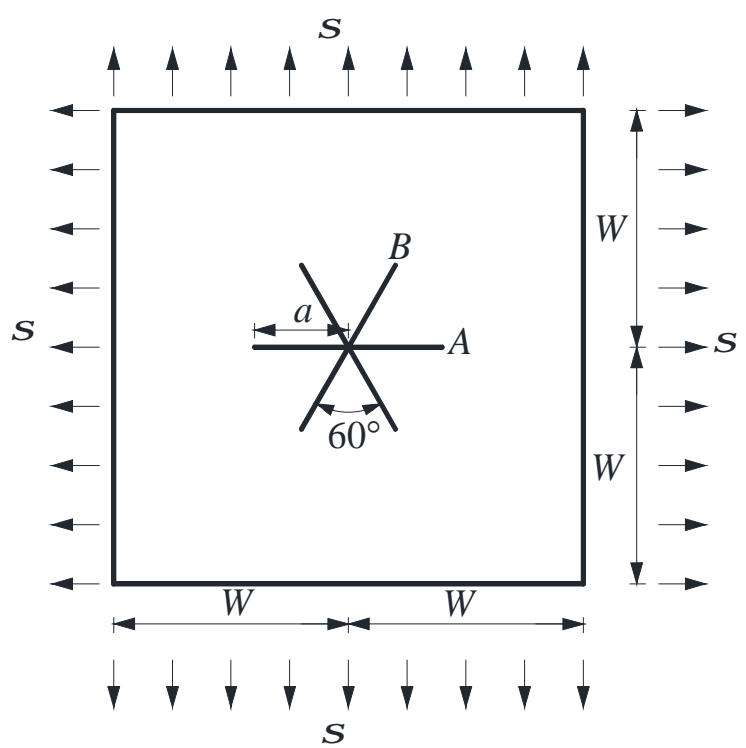

Figure 4. A star-shaped crack in a finite square plate under bi-axial tension.



Figure 5. The mathematical cover adopted for the star-shaped crack when $a / W=0.5$.

The normalized SIFs at crack tips $B$ are defined as follows

$$
\begin{aligned}
& F_{\mathrm{I}}^{B}=K_{\mathrm{I}}^{B} /(\sigma \sqrt{\pi a}) \\
& F_{\mathrm{II}}^{B}=K_{\mathrm{II}}^{B} /(\sigma \sqrt{\pi a})
\end{aligned}
$$

Table 3 Number of physical patches, manifold elements and degrees of freedom for different $a / W$

\begin{tabular}{clllllll}
\hline$a / W$ & 0.2 & 0.3 & 0.4 & 0.5 & 0.6 & 0.7 & 0.8 \\
\hline PPs & 2358 & 2392 & 2420 & 2452 & 2482 & 2510 & 2538 \\
MEs & 4474 & 4508 & 4536 & 4568 & 4598 & 4626 & 4654 \\
DOFs & 14292 & 14496 & 14664 & 14856 & 15036 & 15204 & 15372 \\
\hline
\end{tabular}

In this case, the number of mathematical patches is fixed as 2304, while the numbers of physical patches, manifold elements and the corresponding DOFs are variant with different crack lengths, as shown in Table 3. Obviously, the number of both PPs and MEs will decrease sharply if an adaptive mathematical mesh is selected (Ma et al. 2009).

The normalized SIFs $F_{\mathrm{I}}^{A}$ at crack tip $A, F_{\mathrm{I}}^{B}$ and $F_{\mathrm{II}}^{B}$ at crack tip $B$ are shown in Table 4, 5 and 6 respectively. Similarly, the results by NMM-P and NMM-1 are nearly the same with each other. The results of NMM-P are comparable to the three reference results. 
Table 4 Comparison of normalized SIFs $F_{\mathrm{I}}{ }^{A}$ at crack tip $A$ for a star-shaped crack in a finite plate for various ratios $a / W$

\begin{tabular}{llllll}
\hline$a / W$ & NMM-P & NMM-1 & $*$ & $* *$ & $* * *$ \\
\hline 0.2 & 0.7686 & 0.7597 & 0.7670 & 0.7691 & 0.7570 \\
0.3 & 0.7831 & 0.7808 & 0.7931 & 0.7970 & 0.7846 \\
0.4 & 0.8132 & 0.8113 & 0.8287 & 0.8352 & 0.8255 \\
0.5 & 0.8579 & 0.8556 & 0.8864 & 0.8921 & 0.8852 \\
0.6 & 0.9285 & 0.9266 & 0.9673 & 0.9749 & 0.9758 \\
0.7 & 1.0601 & 1.0577 & 1.0971 & 1.1022 & 1.1142 \\
0.8 & 1.3515 & 1.3492 & 1.3423 & 1.3454 & - \\
\hline
\end{tabular}

*Daux et al. (2000), ${ }^{* *}$ Muravin \& Turkel (2006), ${ }^{* * * C h e u n g ~ e t ~ a l . ~(1992), ~ ' ~-~ ' ~ m e a n s ~ n o ~ c o r r e s p o n d i n g ~ v a l u e ~}$ Table 5 Comparison of normalized SIFs $F_{\mathrm{I}}^{B}$ at crack tip $B$ for a star-shaped crack in a finite plate for various ratios $a / W$

\begin{tabular}{llllll}
\hline$a / W$ & NMM-P & NMM-1 & $*$ & $* *$ & $* * *$ \\
\hline 0.2 & 0.7752 & 0.7696 & 0.7683 & 0.7690 & 0.7578 \\
0.3 & 0.8060 & 0.8043 & 0.7983 & 0.7994 & 0.7884 \\
0.4 & 0.8620 & 0.8602 & 0.8466 & 0.8527 & 0.8365 \\
0.5 & 0.9463 & 0.9453 & 0.9255 & 0.9232 & 0.9088 \\
0.6 & 1.0751 & 1.0741 & 1.0445 & 1.0405 & 1.0182 \\
0.7 & 1.2729 & 1.2722 & 1.2367 & 1.2384 & 1.1936 \\
0.8 & 1.5786 & 1.5798 & 1.5624 & 1.5577 & - \\
\hline
\end{tabular}

Table 6 Comparison of normalized SIFs $F_{\mathrm{II}}^{B}$ at crack tip $B$ for a star-shaped crack in a finite plate for various ratios $a / W$

\begin{tabular}{llllll}
\hline$a / W$ & NMM-P & NMM-1 & $*$ & $* *$ & $* * *$ \\
\hline 0.2 & 0 & 0 & 0.0005 & 0.0007 & 0.00045 \\
0.3 & 0.0020 & 0.0011 & 0.0021 & 0.0020 & 0.00224 \\
0.4 & 0.0058 & 0.0054 & 0.0080 & 0.0077 & 0.0070 \\
0.5 & 0.0166 & 0.0145 & 0.0184 & 0.0201 & 0.0168 \\
0.6 & 0.0321 & 0.0305 & 0.0364 & 0.0451 & 0.0338 \\
0.7 & 0.0561 & 0.0551 & 0.0593 & 0.0622 & 0.0529 \\
0.8 & 0.0869 & 0.0882 & 0.0864 & 0.0804 & - \\
\hline
\end{tabular}

\section{CONCLUSIONS}

The proposed NMM-P is applied to solve the complex crack problems. NMM-P has nearly the same precision with the NMM-1 in even treating the complex cracks such as the crucifix crack and the star-shaped crack. But NMM-P is linearly independent. The results by NMM-P and the reference results are also comparable. Obviously, NMM-P has shown great excellence in even modeling the complex crack problems, which further expands the range of application of NMM-P.

\section{ACKNOWLEDGEMENTS}

The research work of this paper was financially supported by National Natural Science Foundation of China under Project grant No. 11502033, 51579016, 51539002, 51379022.

\section{references}

1) Belytschko, T., Krongauz, Y., Organ, D., Fleming, M. \& Krysl, P. 1996. Meshless methods: an overview and recent developments. Computer Methods in Applied Mechanics and Engineering 139(1): 3-47.

2) Bouchard, P. O., Bay, F., \& Chastel, Y. 2003. Numerical modelling of crack propagation: automatic remeshing and comparison of different criteria. Computer methods in applied mechanics and engineering 192(35): 3887-3908.

3) Cheung, Y. K., Woo, C. W., \& Wang, Y. H. 1992. A general method for multiple crack problems in a finite plate. Computational Mechanics 10(5): 335-343. 
4) Daux, C., Moës, N., Dolbow, J., Sukumar, N., \& Belytschko, T. 2000. Arbitrary branched and intersecting cracks with the extended finite element method. International Journal for Numerical Methods in Engineering 48: 1741-1760.

5) Dolbow, J., \& Belytschko, T. 1999. A finite element method for crack growth without remeshing. International journal for numerical methods in engineering 46(1): 131-150.

6) Ma, G. W., An, X. M., Zhang, H. H., \& Li, L.X. 2009. Modeling complex crack problems using the numerical manifold method. International Journal of Fracture 156(1): 21-35.

7) Melenk, J. M., \& Babuška, I. 1996. The partition of unity finite element method: basic theory and applications. Computer Methods in Applied Mechanics and Engineering 139(1): 289-314.

8) Muravin, B., \& Turkel, E. 2006. Multiple crack weight for solution of multiple interacting cracks by meshless numerical methods. International Journal for Numerical Methods in Engineering 67(8): 1146-1159.

9) Shi, G. H. 1991. Manifold method of material analysis. Transactions of 9th Army Conference on Applied Mathematics and Computing. Minneapolis: Minnesota, 57-76.

10) Strouboulis, T., Babuška, I., \& Copps, K. 2000. The design and analysis of the generalized finite element method. Computer methods in applied mechanics and engineering 181(1): 43-69.

11) Strouboulis, T., Copps, K., \& Babuska, I. 2000. The generalized finite element method: an example of its implementation and illustration of its performance. International Journal for Numerical Methods in Engineering 47(8): 1401-1417.

12) Trädegård, A., Nilsson, F., \& Östlund, S. 1998. FEM-remeshing technique applied to crack growth problems. Computer Methods in Applied Mechanics and Engineering 160(1): 115-131.

13) $\mathrm{Xu}, \mathrm{D}$. D, Zheng, H., Yang, Y. T., \& Wu, A. Q. 2015. Multiple crack propagation based on the numerical manifold method. Chinese Journal of Theoretical and Applied Mechanics 47(3): 471481. (in Chinese)

14) Zhang, H. H., \& Zhang, S. Q. 2012. Extract of stress intensity factors on honeycomb elements by the numerical manifold method. Finite Elements in Analysis and Design 59: 55-65.

15) Zhu, B. F. 1998. The finite element method theory and applications. China Water Power Press, Beijing, 1998. (in Chinese) 